\title{
HÉRNIA DIAFRAGMÁTICA TRAUMÁTICA CRÔNICA E FÍSTULA GASTROPLEUROCUTÂNEA
}

\author{
CHRONIC TRAUMATIC DIAPHRAGMATIC HERNIA AND \\ GASTRICPLEURALCUTANEOUS FISTULA
}

\author{
Marcelo Fernandes Rangel, TCBC-PB ${ }^{1}$ \\ Fernando Antônio de Carvalho ${ }^{2}$ \\ Leonardo Pires de Sá Nóbrega ${ }^{3}$
}

\section{INTRODUÇÃO}

Hérnia diafragmática traumática (HDT) é definida como laceração do diafragma, com passagem de vísceras abdominais para o tórax. De acordo com o período de evolução, pode ser classificada em aguda, latente e crônica. Nesta última fase, a maioria é assintomática, exceto nos casos de complicações obstrutivas, estrangulamento ou necrose das vísceras herniadas.

No período latente, podem ocorrer sintomas vagos, sendo freqüentes os sinais radiológicos sugestivos de afecções pleuropulmonares, como elevação do diafragma, obliteração dos seios costofrênicos, atelectasia pulmonar e derrame pleural, dificultando o diagnóstico e induzindo a erros na conduta terapêutica.

Esta publicação tem por finalidade relatar um caso de paciente portador de HDT crônica, com estômago na cavidade pleural esquerda, cujo diagnóstico radiológico inicial foi de empiema pleural. Submetido à drenagem torácica, houve perfuração do estômago pelo tubo torácico, com estabelecimento de fístula gastropleurocutânea.

\section{RELATO DE CASO}

Paciente masculino, 26 anos, vítima de ferimentos por arma de fogo em tórax e abdome, há cerca de dois anos. Operado em Serviço de Emergência, evoluiu de forma satisfatória durante o referido período, quando veio a apresentar queixas indefinidas, predominantemente torácicas. Procurou atendimento no mesmo serviço, onde foi diagnosticado empiema pleural, e submetido à drenagem fechada do tórax. Após introdução de dieta oral, no pós-operatório, verificou-se eliminação de conteúdo alimentar pelo dreno torácico. Encaminhado ao nosso serviço, realizou-se estudo radiológico contrastado do trato gastrointestinal superior, que demonstrou presença do estômago na cavidade torácica e de dreno de tórax no interior da cavidade gástrica (Figura 1). Foi submetido à correção cirúrgica por acesso abdominal, realizando-se ampliação do anel herniário, redução do estômago para a cavidade abdominal, reparo da lesão gástrica com pontos separados de ácido poliglicólico 3-0 e reparo do defeito diafragmático com polipropileno no 0 , também em pontos separados. Evoluiu sem intercorrências, com alta hospitalar no quinto dia de pós-operatório.

\section{DISCUSSÃO}

A hérnia diafragmática traumática (HDT) é mais comum em indivíduos do sexo masculino, entre a segunda e quarta décadas de vida, quase sempre decorrente de trauma abdominal fechado por acidente automobilístico ${ }^{1}$. O mecanismo implicado é o aumento súbito da pressão intraabdominal em relação à pressão torácica, secundário ao trauma, produzindo um gradiente de pressão no diafragma suficientemente elevado para causar sua ruptura ${ }^{2}$.

Nas HDT, o diafragma esquerdo é acometido em cerda de $90 \%$ dos casos, tendo baixa incidência à direita, pois o fígado dissipa a energia do impacto ${ }^{3}$. O acometimento bilateral ocorre em aproximadamente $1 \%$ dos $\operatorname{casos}^{1}$.

O diagnóstico da HDT pode ser difícil na fase inicial após o trauma, principalmente quando as manifestações clínicas predominantes são secundárias às lesões associadas ${ }^{2}$. Nesses casos, pode ocorrer o encarceramento das vísceras herniadas para o tórax pela lesão do diafragma, caracterizando a HDT crônica.

1. Professor Adjunto Doutor do Departamento de Cirurgia do CCS-UFPB. TCBCD. Ex-Chefe do Serviço de Cirurgia Abdominal do Hospital Napoleão Laureano.

2. Professor Adjunto do Departamento de Cirurgia do CCS-UFPB. Chefe dos Serviços de Cirurgia Torácica do Hospital Napoleão Laureano e do Hospital Universitário da UFPB.

3. Aluno de Graduação do Curso de Medicina do CCS-UFPB. Bolsista do PIBIC/CNPq/UFPB.

Recebido em 20/3/2000

Aceito para publicação em 28/7/2000

Trabalho realizado no Serviços de Cirurgia Abdominal e Torácica do Hospital Napoleão Laureano - João Pessoa-PB. 


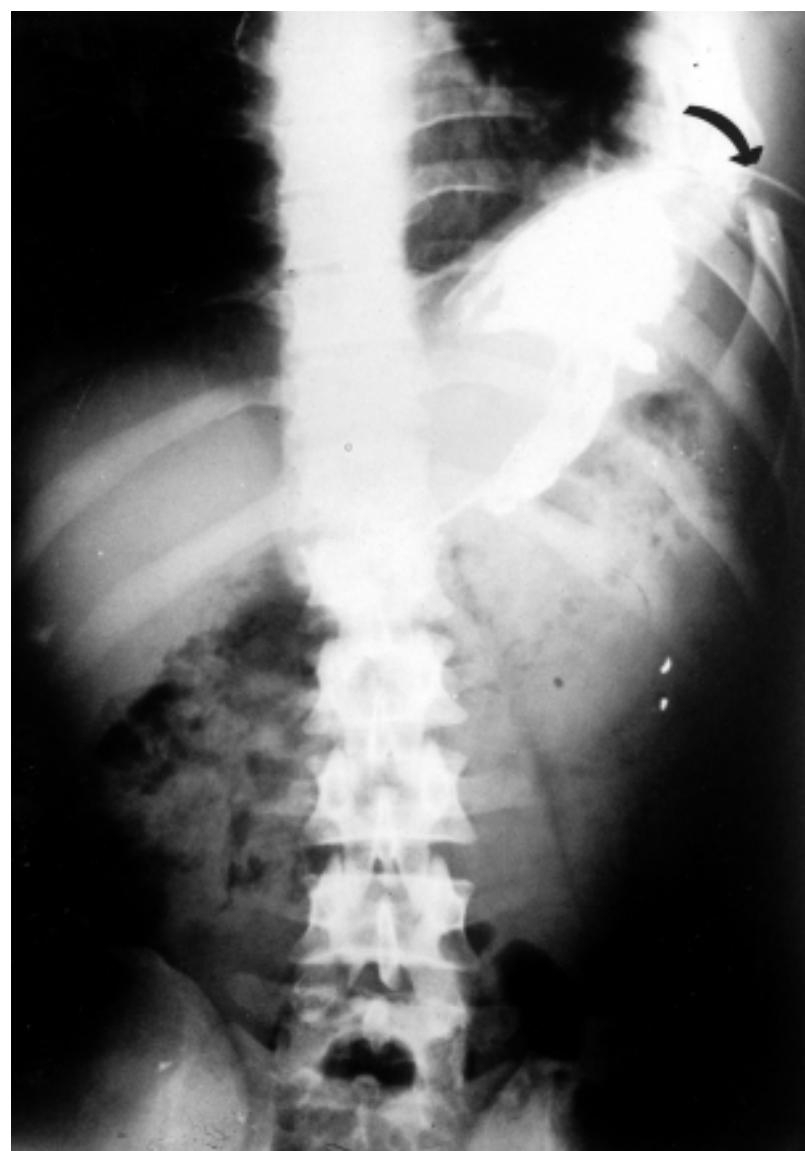

Figura 1 - Radiografia contrastada, mostrando estômago na cavidade torácica, e dreno de tórax no interior da cavidade gástrica (seta).

Os casos de HDT crônica conseqüentes a trauma penetrante são incomuns, pois a laceração diafragmática geralmente é reconhecida durante a exploração cirúrgica, mandatória nos traumatismos penetrantes abdominais ${ }^{4}$.

Mesmo assim, em alguns casos, a lesão diafragmática pode passar despercebida, e as manifestações clínicas só se tornam evidentes meses ou anos após o trauma, con- forme ocorreu no paciente em questão. Desta forma, fica caracterizada a HDT crônica latente, período onde os sintomas são vagos, podendo ser predominantemente torácicos, induzindo a erros no diagnóstico.

Nesses casos, exames contrastados e endoscópicos podem evidenciar a presença de vísceras abdominais no tórax. A ultra-sonografia e a tomografia computadorizada permitem evidenciar a presença dos órgãos herniados, principalmente de vísceras sólidas, quando não demonstradas pela radiologia convencional.

Nos casos de HDT crônica, existe a possibilidade de investigação diagnóstica minuciosa, exceto nos casos complicados por obstrução, estrangulamento, necrose ou perfuração da víscera herniada.

Portanto, condutas intempestivas, como a adotada neste caso, podem condicionar o aparecimento de complicações não diretamente relacionadas à presença da HDT crônica, determinando um aumento na morbi-mortalidade.

O tratamento de HDT é essencialmente cirúrgico. A correção pode ser realizada tanto por acesso abdominal como por toracotomia, na dependência das lesões associadas e do período de evolução ${ }^{4}$, havendo preferência pela laparotomia na fase aguda e pelo acesso torácico nos casos de HDT crônica ${ }^{2}$.

No caso descrito, havia drenagem torácica prévia, o que nos levou a optar pelo acesso abdominal. Apesar da recomendação do acesso torácico, nos casos de HDT crônica $^{2}$, a via de acesso abdominal montrou-se adequada para a correção de ambas as lesões gástrica e diafragmática. Atualmente, o acesso videolaparoscópico tem sido cada vez mais utilizado, permitindo o diagnóstico pela visualização da lesão diafragmática com maior especificidade em relação a outros métodos diagnósticos ${ }^{3}$. Possibilita, ainda, a redução dos órgãos herniados e a correção do defeito diafragmático por meio de sutura primária, utilizando como artifício a diminuição dos níveis de pressão do pneumoperitônio, o que permite a realização da sutura sob menor tensão ${ }^{5}$.

Considerando que a maioria das HDT crônicas é assintomática, é importante ter em mente este diagnóstico, particularmente naqueles pacientes com história de trauma no passado, para que iatrogenias sejam evitadas.

\begin{abstract}
Traumatic diaphragmatic hernia is defined as a laceration of the diaphragm with an abdominal viscera herniation into the thorax. It is usually asymptomatic, with the exception of the cases with obstruction, strangulation, necrosis or perforation of the herniaded viscera. It is classified as acute, latent or chronic, in accordance with the evolutive period. At the latent phase, symptoms are indefinite and the radiological signals, which are suggestive of thoracic affections, are frequent and can induce a diagnosis error, leading to inadequate treatment.This article presents a case of chronic traumatic diaphragmatic hernia which was complicated by a gastricpleuralcutaneous fistula, due to an inadequate thoracic drainage. Considering that this is a chronic affection with an unquestionable surgical indication, due to the complications risk, it is essential to have a detailed diagnostic investigation, which aims at both avoiding an intempestive or inadequate therapeutics behaviour and reducing the affection morbimortality. Recently, the videolaparoscopic approach has proved to be more precise when compared to the other diagnostic methods, by direct visualization of the diaphragmatic laceration, allowing its correction by an immediate suture.
\end{abstract}

Key Words: Chronic traumatic diaphragmatic hernia; Gastric fistula; Pleural empyema. 


\section{REFERÊNCIAS}

1. Zantut LFC, Machado MAC, Volpe P. et al. Bilateral diaphragmatic injury diagnosed by laparoscopy. Rev Paul Med 1993; 111: 430-432.

2. Coelho JCU, Loureiro MP, Andrade RF et al. Oclusão cólica secundária à hérnia diafragmática traumática. Rev Col Bras Cir 1996; 23:227-229.

3. Mccollum C, Anyanwu Ch, Umeh BUD et al, Management of traumatic rupture of diaphragma. Br J Surg 1987; 74: 181-183.

4. Rangel MF, Araújo LB, Pimenta FCF et al. Traumatismos gástricos penetrantes. Rev Col Bras Cir 1992; 19:209212.
5. Domene CE, Volpe P, Birboim I. et al. Correção laparoscópica de hérnia diafragmática traumática crônica. Rev Col Bras Cir 1998; 25: 217-219.

Endereço para correspondência: Dr. Marcelo Fernandes Rangel

Rua Clodoaldo Gouveia, 66 - Centro. 58013-370 - João Pessoa-PB 